\title{
Activation of the PI3K/Akt and MAPK signaling pathways antagonizes adriamycin-induced HL-60 leukemia cell apoptosis
}

\author{
LIPING ZHOU, HONG LUAN, XIHUA DONG and YAN LI \\ Department of Laboratory Medicine, the First Hospital of China Medical University, Liaoning 110001, P.R. China
}

Received February 26, 2010; Accepted May 3, 2010

DOI: $10.3892 / \mathrm{mmr} 00000309$

\begin{abstract}
Adriamycin (ADM) is a drug used in the treatment of various types of cancer and exerts an antineoplastic effect mainly through the induction of apoptosis. Phosphoinositide 3 kinase (PI3K)/Akt and MAPK are fundamental survival pathways activated by exposure to most chemotherapeutical agents. However, the role of these pathways in the ADM-induced apoptosis of leukemia cells remains unclear. In the present study, ADM triggered dose-dependent cytotoxicity and resulted in a significant loss of cell viability in HL-60 cells. Moreover, treatment with ADM significantly reduced mitochondrial membrane potential $\left(\Delta \Psi_{\mathrm{m}}\right)$ in the cells. Akt and ERK activation was also detected, and the inhibition of these two pathways resulted in the enhancement of ADM-induced apoptosis. These results indicate that the PI3K/Akt and ERK survival pathways antagonize the chemotherapeutic effect of ADM. Thus, inhibiting these pathways may serve to enhance the effect of ADM.
\end{abstract}

\section{Introduction}

The anthracycline antitumor agent adriamycin (ADM) is one of the most effective drugs currently available for the treatment of various human neoplastic diseases, including leukemia, lymphomas, sarcomas and breast cancer (1-4). Several mechanisms have been proposed for the antitumor effects of ADM, including DNA intercalation, free radical formation with induction of DNA damage, inhibition of topoisomerase II and activation of apoptosis-related signaling pathways (5-9). However, certain leukemia cells still exhibit insensitivity or resistance to ADM, which results in therapy

Correspondence to: Dr Liping Zhou, Department of Laboratory Medicine, the First Hospital of China Medical University, 155 North Nanjing Street, Heping, Shenyang, Liaoning 110001, P.R. China E-mail: zhouliping750825@hotmail.com

\section{Abbreviations: ADM, adriamycin}

Key words: adriamycin, phosphoinositide 3 kinase/Akt, MAPK, apoptosis, HL-60 cells failure and tumor relapse. Hence, it is important to reveal the resistance mechanisms of these cells.

It has been reported that ADM resistance in solid tumors results not only from the inactivation of apoptotic pathways, but also from the over-activation of survival signals $(10,11)$. The phosphoinositide 3 kinase (PI3K)/Akt pathway is probably the best characterized and most prominent pathway with regard to the transmission of anti-apoptotic signals in cell survival, and is activated by exposure to most chemotherapeutic agents, including ADM and etoposide $(12,13)$, in gastric and breast cancer (14-16). The MEK/ERK1/2 pathway is also known to play a fundamental role in survival, proliferation and apoptosis (17), indicating that the PI3K/Akt and MEK/ ERK pathways have an antagonistic effect on ADM-induced leukemia cell apoptosis.

In the present study, we explored the effects of ADM on HL-60 cell viability and apoptosis, and investigated the regulation and function of the PI3K/Akt and ERK signaling pathways in ADM-induced apoptosis in HL-60 cell lines. The results provide new data regarding how to overcome the resistance of leukemia cells to ADM.

\section{Materials and methods}

Materials and antibodies. 3-(4,5-dimethylthiazol-2-yl)-2,5diphenyl tetrazolium bromide (MTT), dimethyl sulphoxide (DMSO) and the specific PI3K inhibitor LY294002 and MEK/ERK inhibitor PD98059 were from Sigma (St. Louis, MO, USA). Antibody against Cbl-b was from Santa Cruz Biotechnology (Santa Cruz, CA, USA). Antibodies specific to Akt and phospho-Akt (p-Akt) were from Cell Signaling Technology (Danvers, MA, USA). Anti-ERK and pERK anti-tubulin monoclonal antibody was from BD Biosciences Pharmingen (San Jose, CA, USA).

Cell culture. The rat basophilic leukemia cell lines were grown in Dulbecco's modified Eagle's medium (DMEM; Sigma) containing $10 \%$ heat-inactivated fetal calf serum (FCS) in a $37^{\circ} \mathrm{C}$ humidified inbubator with a mixture of $95 \%$ air and $5 \%$ $\mathrm{CO}_{2}$. The cell line was passaged once a week. The cells used in the experiment were obtained from passages 3-7, inoculated in 24-well plates (Nunc, Roskilde, Denmark) at 5x104 cells/well and grown to $85 \%$ confluence in DMEM (DMEM/F12; Sigma) supplemented as above. During the experiments, the cells were detached with $0.05 \%$ trypsin/0.02\% EDTA (Sigma). 
MTT assay. The effects of different agents on cell proliferation were measured using the 3-(4,5-dimethylthiazol-2-yl)-2,5-diphenyltetrazolium bromide (MTT) assay as described previously. Briefly, cells were seeded at $1 \times 10^{4}$ cells/ well in 96-well plates and incubated overnight. Different concentrations of the test agent were then added and incubation was continued for $24 \mathrm{~h}$. Thereafter, $20 \mu \mathrm{l}$ of MTT solution $(5 \mathrm{mg} / \mathrm{ml})$ was added to each well and the cells were incubated for a further $4 \mathrm{~h}$ at $37^{\circ} \mathrm{C}$. After removal of the culture medium, the cells were lysed in $150 \mu \mathrm{l}$ of DMSO and the optical density (OD) was measured at $570 \mathrm{~nm}$ using a microplate reader (Bio-Rad, Hercules, CA, USA). The following formula was used: cell viability $=(\mathrm{OD}$ of the experimental sample $/ \mathrm{OD}$ of the control group) $\mathrm{x} 100 \%$.

Western blot analysis. Total cellular proteins were extracted and quantified as previously described. Aliquots $(50 \mu \mathrm{g})$ of each lysate were electrophoresed on SDS-PAGE gels and then blotted onto nitrocellulose membranes. The membranes were blocked with $5 \%$ nonfat milk in TBST $(10 \mathrm{mM}$ Tris- $\mathrm{HCl}$, $\mathrm{pH} 7.4,100 \mathrm{mM} \mathrm{NaCl}, 0.5 \%$ Tween-20) for $2 \mathrm{~h}$ at room temperature, then incubated overnight at $4^{\circ} \mathrm{C}$ in $5 \%$ non-fat milk in TBST containing either p-Akt, Akt, Cbl-b, p-ERK, ERK or tubulin antibodies, followed by a 1-h incubation with the peroxidase-conjugated second antibody. After extensive washing in TBST, proteins were visualized using enhanced chemiluminescence reagent (SuperSignal Western Pico Chemiluminescent Substrate; Pierce, Rockford, IL, USA). The results were analyzed by NIH Image J software.

Flow cytometry. Cells were cultured in the presence or absence of DNR or EPI for the indicated times, then harvested and fixed with ice-cold $70 \%(\mathrm{v} / \mathrm{v})$ ethanol for $24 \mathrm{~h}$. After centrifugation at $200 \mathrm{x} \mathrm{g}$ for $5 \mathrm{~min}$, the cell pellet was washed with PBS ( $\mathrm{pH}$ 7.4) and resuspended in PBS containing propidium iodide $(10 \mu \mathrm{g} / \mathrm{ml})$ and DNase-free RNase $(20 \mu \mathrm{g} / \mathrm{ml})$. Cells were then incubated at room temperature in the dark for $30 \mathrm{~min}$, and DNA content was determined by flow cytometry using a FACScan flow cytometer (Becton Dickinson, San Jose, CA, USA). Data were analyzed using MODIFIT software (Becton Dickinson) (20).

Statistical analysis. Data are presented as the means \pm standard deviation (SD). The significance of the difference between the groups was assessed by the Student's two-tailed t-test. P-values of $<0.05$ were considered significant. Means were calculated from at least three independent experiments.

\section{Results}

Effects of ADM on HL-60 cell viability and apoptosis. To investigate the effects of ADM on cell viability, HL-60 cells were treated with the indicated concentrations of ADM for $24 \mathrm{~h}$. As confirmed by the MTT assay, ADM triggered dosedependent cytotoxicity and resulted in a significant loss of cell viability (Fig. 1A). Apoptosis was measured after exposure to $1 \mu \mathrm{M}$ ADM for the indicated times. As shown in Fig. 1B, apoptosis was induced in a time-dependent manner. The percentage of apoptotic cells at $24 \mathrm{~h}$ was $31 \%$. These results indicate that the influence of ADM on the inhibition of the
A

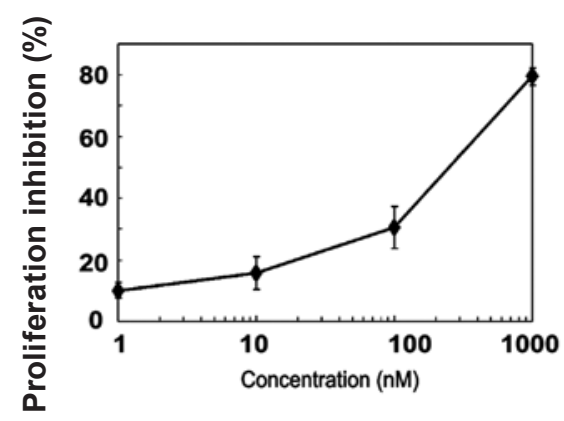

B

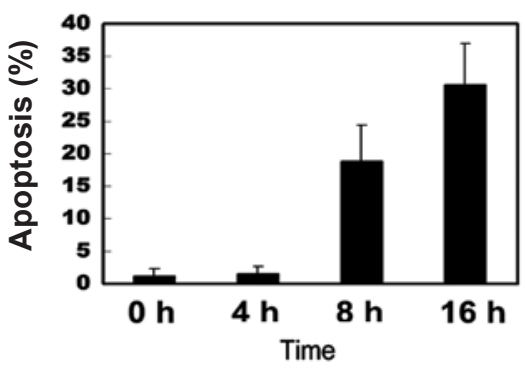

Figure 1. Effects of ADM on HL-60 cell viability and apoptosis. (A) Percentage of proliferation inhibition in relation to cytotoxicity with different concentrations of ADM. A significant loss of cell viability was observed. (B) Percentage of apoptotic cells at different time points. The percentage of apoptotic cells increased in a time-dependent manner. Results are representative of at least three independent experiments.

proliferation of HL-60 cells in vitro mainly results from apoptotic cell death in a time-dependent manner.

Mitochondrial membrane potential $\left(\Delta \Psi_{m}\right)$ reduction and caspase-3 activtion during ADM-induced apoptosis. In many systems, apoptosis is associated with the loss of $\Delta \Psi_{\mathrm{m}}$, which may be regarded as a limiting factor in the apoptotic pathway (18). To investigate the effect of $\Delta \Psi_{\mathrm{m}}$ in ADM-induced apoptosis, cells were stained with DiOC6, a mitochondria-specific and voltage-dependent dye. Treatment of the HL-60 cells with ADM significantly reduced their $\Delta \Psi_{\mathrm{m}}$ (Fig. 2A). When exposed to ADM and incubated for $12 \mathrm{~h}$, the percentage of apoptotic cells in the samples evidenced a marked increase $(47.6 \%)$ as compared to the control (5.9\%) (Fig. 2A). It is known that caspase-3 functions during the later steps of the protease cascade, and that the activation of caspase- 3 is linked with apoptosis through a mitochondrial function. Therefore, we further examined caspase-3-related apoptotic signals in ADM-treated HL-60 cells. As shown in Fig. 2B, ADM resulted in the activation of caspase- 3 by Western blot analysis and maximal caspase- 3 activation occurred at $16 \mathrm{~h}$. As shown in Fig. 2, the alteration of $\Delta \Psi_{m}$ is correlated with that of caspase-3 activation during ADM-induced apoptosis.

Effects of ADM on Akt and ERK activation. To investigate whether the PI3K/Akt and ERK pathways are involved in the regulation of ADM chemosensitivity, HL-60 cells were treated with ADM for 0,8 and $16 \mathrm{~h}$ and Western blotting was performed. A significant degree of Akt activation was detected. As shown in Fig. 3A, maximal increases in Akt 
A
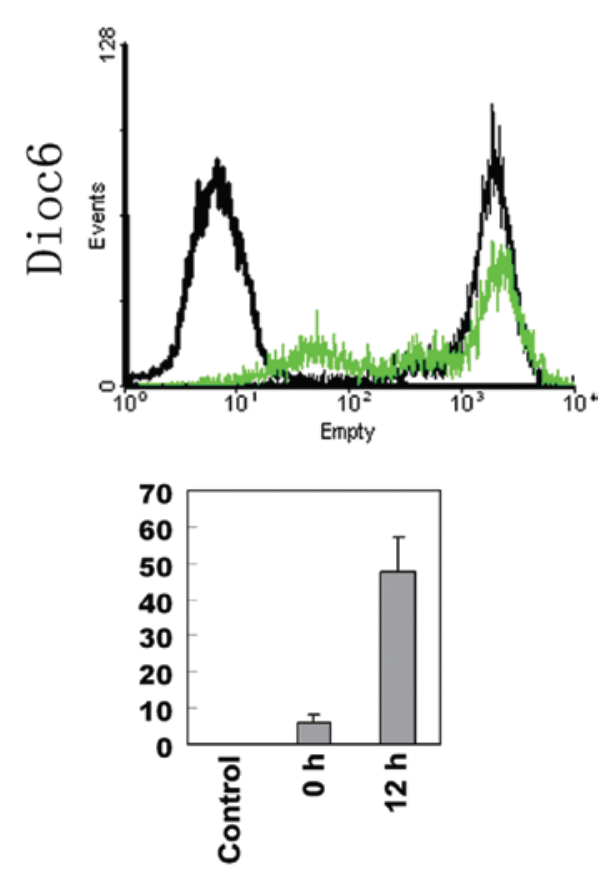

B

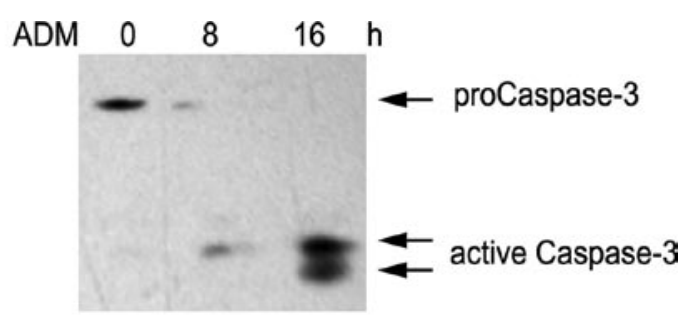

IB: Anti-Caspase-3

Figure 2. (A) Changes in mitochondria membrane potential in relation to ADM-induced apoptosis. Cells were stained with Dioc6 and analyzed using FCM at 0 and $12 \mathrm{~h}$. The results are summarized in a column graph. (B) HL-60 cells were exposed to $100 \mathrm{nM}$ ADM for 0,8 and $16 \mathrm{~h}$, then cell lysates were seperated using SDS-PAGE and blotted with anti-caspase-3 antibody. Results are representative of at least three independent experiments.

phosphorylation occurred at $8 \mathrm{~h}$, after which the levels of p-Akt protein gradually declined and reached basal levels at $16 \mathrm{~h}$. By contrast, the protein levels of total Akt were not altered (Fig. 3A). On the other hand, ERK activation was slower than that of Akt, reaching the peak value at $16 \mathrm{~h}$. These results indicate that both Akt and ERK activation may result in resistance to ADM (Fig. 3B).

Effects of PI3K/Akt and ERKs on ADM-induced apoptosis. To characterize the relative roles of Akt and ERK on ADM-induced apoptosis, two different selective inhibitors (LY294002 for the PI3K pathway and PD98059 for the MEK/ERK pathway) were used. ADM alone did not induce HL-60 cell apoptosis, while ADM plus the PI3K/Akt inhibitor LY294002 significantly increased apoptosis to $72.1 \%$ (Fig. 3C). Notably, cells co-treated with ADM and the ERK inhibitor exhibited similar synergetic effects (Fig. 3C). Our results strongly suggest that the inactivation of PI3K/Akt may play an important role in ADM-induced apoptosis. Therefore, the activation of Akt and ERK is functionally involved in the negative regulation of ADM-induced apoptosis in HL-60 cells.
A

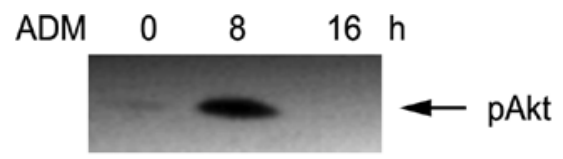

IB: Anti-pAkt

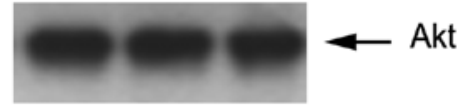

IB: Anti-Akt

B

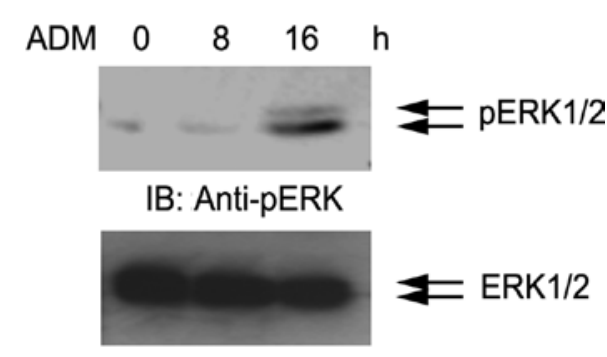

IB: Anti-ERK

C

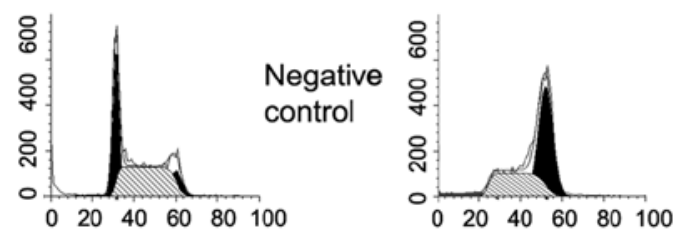

ADM
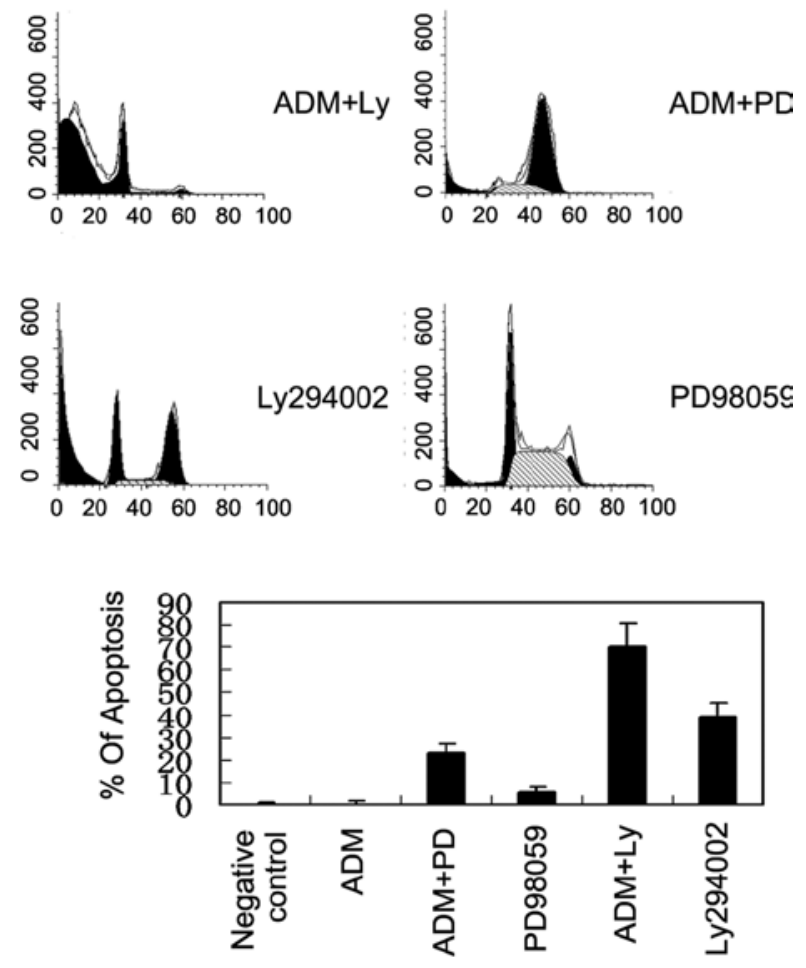

Figure 3. (A) Effects of ADM on Akt activation. (A and B) pAkt protein levels. HL-60 cells were treated with ADM for 0,8 and $16 \mathrm{~h}$, then cell lysates were separated using SDS-PAGE and blotted with anti p-Akt and Akt (A) or anti-pERK1/2 and ERK1/2 (B) antibodies. (C) Effects of PI3K/Akt and ERK on ADM-induced apoptosis. HL-60 cells were treated with the indicated reagents, then cells were stained with propidine iodide (PI). Apoptosis was analyzed using FCM. The results are summarized in the column graph. Results are representative of at least three independent experiments. 


\section{Discussion}

The successful treatment of acute myeloid leukemia (AML) is frequently impeded by the development of resistance to a wide spectrum of cytotoxic drugs. Previous studies have shown that ADM resistance is a consequence of the failure of leukemic cells to engage apoptosis $(19,20)$. Apoptosis is an energy-requiring suicide programme that is normally activated in response to cellular damage (21). The morphological and physiological events of the apoptotic process include the loss of mitochondrial membrane potential, the activation of caspases, PARP cleavage, nuclear DNA fragmentation and cellular shrinkage $(22,23)$. In this study, we found that the treatment of HL-60 cells with ADM for $12 \mathrm{~h}$ significantly reduced their $\Delta \Psi_{\mathrm{m}}$, while the percentage of apoptotic cells in the samples was markedly increased $(47.6 \%)$ as compared to the control (5.9\%). Caspase-3 activation was also detected. These results indicate that ADM induced HL-60 cell apoptosis through mitochondria-mediated pathways.

The PI3K/Akt pathway represents the most relevant survival pathway involved in chemotherapy resistance in solid cancer cells. Down-regulation by means of PI3K inhibitors lowers resistance to various types of therapy in tumor cell lines. In the present study, Akt was strongly activated by ADM from $8 \mathrm{~h}$. The PI3K specific inhibitor LY294002 significantly enhanced ADM-induced apoptosis (Fig. 3C). These results indicate that the PI3K/Akt survival pathway has an antagonistic effect on the chemosensitivity of cells to ADM. Thus, the inhibition of this pathway serves to promote the effect of ADM.

The activation of the ERK1/2 pathway is normally associated with cell proliferation and survival. However, recent studies have indicated that ERK1/2 may exert a dual effect on proliferation. Few studies have focused on the regulation and function of ERK1/2 in ADM-induced cell death (24-26). ADM has been determined to induce the activation of ERK1/2 in neuroblastoma SKN-SH cells, and ERK1/2 activation in the cells appears to play an important role in the enhancement of apoptosis. Our study consistently demonstrated that ADM-induced apoptosis increased with sustained ERK1/2 activation. The suppression of ERK resulted in a significant synergistic effect on apoptosis, such that the degree of apoptosis was much higher than that observed with ADM or ERK inhibitor (PD98059) treatment alone.

In conclusion, this study suggests that both Akt and ERK activation may be associated with the suppression of ADM-induced apoptosis. Our findings elucidate the signal transduction pathways associated with the induction of apoptosis.

\section{References}

1. Wiernik PH and Dutcher JP: Clinical importance of anthracyclines in the treatment of acute myeloid leukemia. Leukemia 6 : 67-69, 1992.

2. Lown JW: Anthracycline and anthraquinone anticancer agents: current status and recent developments. Pharmacol Ther 60: 185-214, 1993.

3. Martelli AM, Nyåkern M, Tabellini G, et al: Phosphoinositide 3-kinase/Akt signaling pathway and its therapeutical implications for human acute myeloid leukemia. Leukemia 20: 911-928, 2006.

4. Zhao Y, You H, Yang Y, et al: Distinctive regulation and function of PI 3K/Akt and MAPKs in doxorubicin-induced apoptosis of human lung adenocarcinoma cells. J Cell Biochem 91: 621-632, 2004.
5. Kostrzewa-Nowak D, Paine MJ, Wolf CR and Tarasiuk J: The role of bioreductive activation of doxorubicin in cytotoxic activity against leukaemia HL 60-sensitive cell line and its multidrug-resistant sublines. Br J Cancer 93: 89-97, 2005.

6. Opel D, Westhoff MA, Bender A, et al: Phosphatidylinositol 3-kinase inhibition broadly sensitizes glioblastoma cells to death receptor and drug-induced apoptosis. Cancer Res 68: 6271-6280, 2008.

7. Gewirtz DA: A critical evaluation of the mechanisms of action proposed for the antitumor effects of the anthracycline antibiotics adriamycin and daunorubicin. Biochem Pharmacol 57: 727-741, 1999.

8. Wu CH, Gordon J, Rastegar M, et al: Proteinase-3, a serine protease which mediates doxorubicin-induced apoptosis in the HL-60 leukemia cell line, is downregulated in its doxorubicinresistant variant. Oncogene 21: 5160-5174, 2002.

9. Mizutani H, Tada-Oikawa S, Hiraku Y, et al: Mechanism of apoptosis induced by doxorubicin through the generation of hydrogen peroxide. Life Sci 76: 1439-1453, 2005.

10. Anzaldi M, Macciò C, Mazzei M, et al: Antiproliferative and proapoptotic activities of a new class of pyrazole derivatives in HL-60 cells. Chem Biodivers 6: 1674-1687, 2009.

11. Lee ER, Kim JY, Kang YJ, et al: Interplay between PI3K/Akt and MAPK signaling pathways in DNA-damaging drug-induced apoptosis. Biochim Biophys Acta 1763: 958-968, 2006.

12. Damrot J, Nübel T, Epe B, et al: Lovastatin protects human endothelial cells from the genotoxic and cytotoxic effects of the anticancer drugs doxorubicin and etoposide. Br J Pharmacol 149: 988-997, 2006

13. Choi SK, Seo BR, Lee KW, et al: Saucernetin-7 isolated from Saururus chinensis induces caspase-dependent apoptosis in human promyelocytic leukemia HL-60 cells. Biol Pharm Bull 30: 1516-1522, 2007.

14. Cho HS, Chang SH, Chung YS, et al: Synergistic effect of ERK inhibition on tetrandrine-induced apoptosis in A549 human lung carcinoma cells. J Vet Sci 10: 23-28, 2009.

15. Oktem G, Karabulut B, Selvi N, et al: Differential effects of doxorubicin and docetaxel on nitric oxide production and inducible nitric oxide synthase expression in MCF-7 human breast cancer cells. Oncol Res 14: 381-386, 2004.

16. Yu HG, Ai YW, Yu LL, et al: Phosphoinositide 3-kinase/Akt pathway plays an important role in chemoresistance of gastric cancer cells against etoposide and doxorubicin induced cell death. Int J Cancer 122: 433-443, 2008.

17. Widmann C, Gibson S, Jarpe MB and Johnson GL: Mitogenactivated protein kinase: conservation of a three-kinase module from yeast to human. Physiol Rev 79: 143-180, 1999.

18. Lee DH, Park T and Kim HW: Induction of apoptosis by disturbing mitochondrial-membrane potential and cleaving PARP in jurkat $\mathrm{T}$ cells through treatment with acetoxyscirpenol mycotoxins. Biol Pharm Bull 29: 648-654, 2006.

19. Jesse P, Mottke G, Eberle J, et al: Apoptosis-inducing activity of Helleborus niger in ALL and AML. Blood Cancer 52: 464-469, 2009.

20. West KA, Castillo SS and Dennis PA: Activation of the PI3K/ Akt pathway and chemotherapeutic resistance. Drug Resistance 5: 234-248, 2002.

21. Lugli E, Troiano L, Ferraresi R, et al: Characterization of cells with different mitochondrial membrane potential during apoptosis. Cytometry A 68: 28-35, 2005.

22. Tembe $\mathrm{V}$ and Henderson BR: BARD1 translocation to mitochondria correlates with Bax oligomerization, loss of mitochondrial membrane potential and apoptosis. J Biol Chem 282: 20513-20522, 2007.

23. Nasser MW, Datta J, Nuovo G, et al: Down-regulation of micro-RNA-1 (miR-1) in lung cancer. Suppression of tumorigenic property of lung cancer cells and their sensitization to doxorubicin-induced apoptosis by miR-1. J Biol Chem 283: 33394-333405, 2008.

24. Liu J, Mao W, Ding B and Liang CS: ERKs/p53 signal transduction pathway is involved in doxorubicin-induced apoptosis in H9c2 cells and cardiomyocytes. Am J Physiol Heart Circ Physiol 295: H1956-H1965, 2008.

25. Kang YJ, Zhou ZX, Wang GW, et al: Suppression by metallothionein of doxorubicin-induced cardiomyocyte apoptosis through inhibition of p38 mitogen-activated protein kinases. J Biol Chem 275: 13690-13698, 2000.

26. Guise S, Braguer D, Carles G, et al: Hyperphosphorylation of tau is mediated by ERK activation during anticancer drug induced apoptosis in neuroblastoma cells. J Neurosci Res 63: 257-267, 2001. 